\title{
High-frequency miniprobe endoscopic ultrasonography in the management of benign esophageal strictures
}

\author{
Surinder Singh Rana a, Ravi Sharmaa, Kamal Kishore ${ }^{b}$, Rajesh Gupta \\ Postgraduate Institute of Medical Education and Research (PGIMER), Chandigarh, India
}

\begin{abstract}
Background Endoscopic ultrasonography (EUS) can predict the response to endoscopic dilatation by delineating the extent of esophageal wall involvement in benign strictures. In contrast to conventional echoendoscopes, the EUS miniprobe can be negotiated across the stricture and thus provide more information. This study retrospectively evaluated the role of miniprobe EUS in predicting the response to endoscopic dilatation in benign esophageal strictures.
\end{abstract}

Methods We analyzed the records of 24 patients (mean age: $48.1 \pm 17.9$ years) with benign esophageal strictures (corrosive 11 , peptic 5, post-radiation 3 , anastomotic 2 , and others 3 ) who underwent miniprobe EUS prior to endoscopic dilatation.

Results The stricture was located in the upper, middle and lower esophagus in 2, 9 and 13 patients, respectively. The mean length of the stricture was $3.4 \pm 1.9 \mathrm{~cm}$. Miniprobe EUS was able to examine the stricture completely in all patients. The mucosa was involved in 6 , mucosa and submucosa in 4 , and mucosa, submucosa and muscularis propria in 14 patients. The mean maximum wall thickness of esophageal wall on EUS at the level of the stricture was $8.2 \pm 2.8 \mathrm{~mm}$. The mean number of sessions required to achieve adequate dilation was $4.7 \pm 2.6$. Patients with mucosal involvement required significantly fewer endoscopic sessions for adequate dilatation as compared to patients with muscularis propria involvement ( 1.8 vs. 6.2 sessions, respectively; $\mathrm{P}=0.0002)$. Patients with greater esophageal wall thickness required more endoscopic sessions $(\mathrm{r}=0.737)(\mathrm{P}=0.00004)$.

Conclusion Miniprobe EUS, by delineating the extent of wall involvement as well as measuring wall thickness in benign esophageal strictures, can predict the response to endoscopic dilatation.

Keywords Endosonography, stricture, dilatation, corrosive, peptic stricture

Ann Gastroenterol 2020; 33 (1): 1-5

\section{Introduction}

Benign esophageal strictures are an important cause of mechanical dysphagia and are caused by various etiologies, including acid reflux (peptic), corrosive or drug-induced, post-surgical (anastomotic), eosinophilic esophagitis, postendoscopic submucosal dissection, and post-radiotherapyinduced $[1,2]$. With the advent of minimally invasive as

Department of a Gastroenterology (Surinder Singh Rana, Ravi Sharma), biostatistics (Kamal Kishore). 'Surgery (Rajesh Gupta), Postgraduate Institute of Medical Education and Research (PGIMER), Chandigarh, India

Conflict of Interest: None

Correspondence to: Dr Surinder Singh Rana, Professor, Department of Gastroenterology, PGIMER, Chandigarh 160 012, India,

e-mail: drsurinderrana@gmail.com

Received 26 August 2019; accepted 12 November 2019; published online 29 November 2019

DOI: https://doi.org/10.20524/aog.2019.0436 well as effective endoscopic bougie or balloon dilatation, endoscopic dilatation has become the procedure of choice in the management of dysphagia due to benign esophageal strictures $[2,3]$. Up to $90 \%$ of benign esophageal strictures can be successfully treated with 1-3 sessions of endoscopic dilatation, with good-long term outcomes $[2,3]$. However, $30-40 \%$ of patients successfully managed with endoscopic dilatation tend to have recurrence of symptoms within the first year of follow up [2,4]. Moreover, complex esophageal strictures that are longer $(>2 \mathrm{~cm})$, irregular, or angulated with a tight stenotic lumen require multiple sessions of dilatation and also show a higher frequency of recurrence $[5,6]$.

Studies have attempted to determine factors that can predict the response to endoscopic dilatation. Patients with peptic strictures usually require fewer sessions of endoscopic dilatation in comparison to patients with corrosive and radiation-induced esophageal strictures [2,7-9]. It has been hypothesized that this difference could be due to the degree of fibrosis of the esophageal wall, as increased fibrosis in the wall has been demonstrated in the histopathology of the resected esophagus in patients with corrosive strictures [10]. Lahoti 
et al have shown that the response to endoscopic dilatation can be predicted by measuring the maximal esophageal wall thickness on computed tomography, with patients having wall thickness of $9 \mathrm{~mm}$ or more requiring a significantly higher number of sessions $(7.57 \pm 1.80$ vs. $1.42 \pm 0.27 ; \mathrm{P}<0.05)$. This is the result given in the quoted study [10]. We had previously shown that endoscopic ultrasound (EUS) provides more detailed information about the esophageal wall, including the wall thickness and the extent of involvement, in patients with benign esophageal strictures $[11,12]$. The information about the extent of involvement of esophageal wall predicts the response to dilatation: patients with involvement of muscularis propria require more dilatation sessions than patients with involvement of mucosa and submucosa only [12]. However, in that earlier study we used a radial echoendosocope, not negotiable across the esophageal stricture; thus, the EUS examination was performed from the proximal end of the stricture. This led to incomplete EUS examination and could have understaged the extent of involvement of the esophageal wall in some patients.

Therefore, we conducted the current study using highfrequency EUS miniprobes that could be negotiated across the stricture, so that a complete EUS evaluation of the stricture could be achieved. We retrospectively evaluated patients with benign esophageal strictures who had undergone miniprobe EUS prior to endoscopic dilatation and evaluated the EUS parameters that could predict response to dilatation.

\section{Patients and methods}

This retrospective study was conducted at a large tertiary care hospital in north India and included all patients with benign esophageal strictures who underwent miniprobe EUS prior to endoscopic dilatation over a 53-month period (December 2014 to May 2019). The clinical and imaging details, and the miniprobe EUS findings were retrieved from the database and analyzed in relation to the details and outcome of the endoscopic dilatation.

All the enrolled patients had significant dysphagia requiring esophageal dilation. The strictures were evaluated in detail by barium studies and upper gastrointestinal endoscopy prior to dilatation. The etiology of the esophageal stricture was determined by a combination of clinical history and endoscopic, radiological and histopathological features. Informed consent was obtained from each patient prior to miniprobe EUS examination as well as endoscopic dilatation. The dysphagia was graded on a scale of 0 to 4 as follows: 0 , able to take normal diet; 1 , unable to swallow certain solids; 2 , able to swallow only semisolid soft diet; 3 , able to swallow liquids only; and 4, unable to swallow even liquids in adequate amounts [13].

\section{Miniprobe EUS examination}

All the patients initially underwent upper gastrointestinal endoscopy and the esophageal stricture was identified.
Thereafter, miniprobe EUS examination was performed using a high-frequency 3-dimensional (3D) catheter probe (20 MHz miniprobe, UM-DG20-31R; Olympus, Tokyo, Japan) with radial, linear and oblique $3 \mathrm{D}$ reconstructions, as we have described previously [14]. Briefly, the procedure was performed with the patient under conscious sedation using intravenous midazolam. The miniprobe was inserted through the instrument channel and negotiated across the esophageal stricture under endoscopic vision. If it was not possible to pass the miniprobe across the stricture blindly, it was inserted over the guide wire without the use of fluoroscopy. The EUS examination was performed at multiple levels of the stricture by gradually withdrawing the probe. The EUS parameters assessed were wall stratification and maximum wall thickness. The EUS findings at the proximal end of the stricture were specifically recorded and compared with the EUS findings obtained at other levels of the stricture.

After performing miniprobe EUS examination, patients underwent endoscopic bougie dilatation using Savary-Gilliard polyvinyl dilators $(7,9,11,12.8,14$ and $15 \mathrm{~mm}$, Wilson-Cook Medical Inc., Winston-Salem, NC). The endoscopic dilation was done at 3-weekly intervals until dilatation up to $15 \mathrm{~mm}$ was achieved and there was complete relief of dysphagia. The number of sessions required to achieve adequate dilation was retrieved from the database. Complications, if any, of endoscopic dilatation, as well as any need for surgery were also retrieved from the database. Thereafter, patients were on regular follow up and dilation was repeated whenever the patient experienced recurrence of dysphagia.

\section{Results}

The records of 24 patients (16 male; mean age: $48.1 \pm 17.9$ years) with benign esophageal strictures (corrosive 11 , peptic 5 , post-radiation 3 , anastomotic 2 , and others 3) who underwent miniprobe EUS prior to endoscopic dilatation were retrieved and analyzed (Table 1). The stricture was located in the upper, middle and lower esophagus in 2, 9 and 13 patients, respectively. The corrosive stricture was due to acid ingestion in 8 patients and alkali ingestion in 3 patients. Six patients had Grade II dysphagia and 18 patients had Grade III dysphagia. The mean length of the strictures was $3.4 \pm 1.9 \mathrm{~cm}$.

Miniprobe EUS was able to completely examine the stricture in all the patients, with no need for prior dilatation. The mucosa was involved in 6 , mucosa and submucosa in 4 , and mucosa, submucosa and muscularis propria in 14 patients (Fig. 1-4). The maximum mean wall thickness of the esophageal wall on EUS at the level of stricture was $8.2 \pm 2.8 \mathrm{~mm}$. The maximum esophageal wall thickness was significantly greater in patients with corrosive strictures than in patients with peptic strictures $(9.5 \pm 2.6 \mathrm{~mm}$ vs. $4.8 \pm 0.7 \mathrm{~mm}$; $\mathrm{P}=0.001)$. In patients with a peptic stricture, the mucosal thickness involved only the mucosa $(n=5)$ and in none of the patients was either the submucosa or the muscularis propria involved. However, the muscularis propria was involved in $8 / 11(72.7 \%)$ patients with corrosive strictures. In the remaining 3 patients with corrosive 
Table 1 Profile of patients with esophageal stricture

\begin{tabular}{|c|c|c|c|c|c|c|c|c|c|c|}
\hline Sr. No. & $\begin{array}{c}\text { Age } \\
\text { (years) }\end{array}$ & Sex & $\begin{array}{l}\text { Grade of } \\
\text { dysphagia }\end{array}$ & $\begin{array}{l}\text { Depth of } \\
\text { involvement }\end{array}$ & Etiology & $\begin{array}{l}\text { Number of } \\
\text { dilatations }\end{array}$ & Recurrence & Surgery & $\begin{array}{l}\text { Length of } \\
\text { stricture } \\
(\mathrm{cm})\end{array}$ & $\begin{array}{l}\text { Wall } \\
\text { thickness } \\
(\mathrm{mm})\end{array}$ \\
\hline 1 & 62 & Male & III & $\mathrm{M}$ & Peptic & 2 & No & No & 1.4 & 4.2 \\
\hline 2 & 71 & Male & II & M & Peptic & 1 & No & No & 1.2 & 3.9 \\
\hline 3 & 68 & Female & II & M & Peptic & 3 & No & No & 1.6 & 5.1 \\
\hline 4 & 54 & Male & II & M & Miscellaneous & 1 & No & No & 0.8 & 4.5 \\
\hline 5 & 28 & Female & III & $\mathrm{M} / \mathrm{SM}$ & Corrosive & 5 & No & No & 3.4 & 6.8 \\
\hline 6 & 62 & Male & III & $\mathrm{M} / \mathrm{SM} / \mathrm{MP}$ & Miscellaneous & 7 & Yes & No & 0.8 & 9.9 \\
\hline 7 & 59 & Male & II & M & Peptic & 2 & No & No & 1.9 & 4.9 \\
\hline 8 & 71 & Male & III & $\mathrm{M} / \mathrm{SM} / \mathrm{MP}$ & Radiation & 4 & No & No & 1.3 & 8.9 \\
\hline 9 & 38 & Male & III & $\mathrm{M} / \mathrm{SM} / \mathrm{MP}$ & Corrosive & 9 & No & No & 6.2 & 12.2 \\
\hline 10 & 28 & Female & III & $\mathrm{M} / \mathrm{SM} / \mathrm{MP}$ & Corrosive & 3 & No & Yes & 7.1 & 11.8 \\
\hline 11 & 70 & Male & III & $\mathrm{M} / \mathrm{SM} / \mathrm{MP}$ & Radiation & 6 & No & No & 3.2 & 10.3 \\
\hline 12 & 60 & Male & III & $\mathrm{M} / \mathrm{SM} / \mathrm{MP}$ & Miscellaneous & 3 & Yes & Yes & 4.8 & 11.2 \\
\hline 13 & 42 & Female & II & $\mathrm{M} / \mathrm{SM}$ & Miscellaneous & 3 & No & No & 1.8 & 5.2 \\
\hline 14 & 38 & Female & III & $\mathrm{M} / \mathrm{SM} / \mathrm{MP}$ & Corrosive & 9 & No & No & 4.9 & 13.2 \\
\hline 15 & 28 & Male & III & $\mathrm{M} / \mathrm{SM} / \mathrm{MP}$ & Corrosive & 7 & Yes & Yes & 7.1 & 12.9 \\
\hline 16 & 21 & Female & II & $\mathrm{M} / \mathrm{SM}$ & Corrosive & 5 & No & No & 4.1 & 7.1 \\
\hline 17 & 31 & Female & III & $\mathrm{M} / \mathrm{SM}$ & Corrosive & 3 & No & No & 3.9 & 6.2 \\
\hline 18 & 27 & Female & III & $\mathrm{M} / \mathrm{SM} / \mathrm{MP}$ & Corrosive & 7 & No & No & 6.2 & 9.1 \\
\hline 19 & 39 & Male & III & $\mathrm{M} / \mathrm{SM} / \mathrm{MP}$ & Corrosive & 11 & No & No & 4.9 & 11.3 \\
\hline 20 & 71 & Male & III & M & Peptic & 2 & No & No & 2.7 & 6.1 \\
\hline 21 & 24 & Male & III & $\mathrm{M} / \mathrm{SM} / \mathrm{MP}$ & Corrosive & 5 & No & No & 4.7 & 7.3 \\
\hline 22 & 62 & Male & III & $\mathrm{M} / \mathrm{SM} / \mathrm{MP}$ & Miscellaneous & 7 & No & No & 1.3 & 9.7 \\
\hline 23 & 71 & Male & III & $\mathrm{M} / \mathrm{SM} / \mathrm{MP}$ & Radiation & 3 & No & No & 4.2 & 9.1 \\
\hline 24 & 30 & Male & III & $\mathrm{M} / \mathrm{SM} / \mathrm{MP}$ & Corrosive & 6 & No & No & 3.1 & 7.2 \\
\hline
\end{tabular}

M, mucosa; SM, submucosa; $M P$, muscularis propria

strictures, the muscularis propria was spared and only the mucosa and submucosa were involved.

The EUS findings obtained from the proximal end of the stricture were also compared with the EUS findings obtained from the remaining part of the stricture. Four patients had involvement of both mucosa and submucosa. However, EUS images from the proximal end of the stricture in these patients revealed involvement of mucosa in only $2 / 4(50 \%)$ patients. Similarly, 14 patients had involvement of muscularis propria, and EUS images from the proximal end of the stricture in these patients revealed involvement of mucosa in only 2 patients, mucosa and submucosa in 3 patients, and muscularis propria involvement in 9 patients.

The mean number of sessions required to achieve adequate dilation was $4.7 \pm 2.6$. Patients with mucosal involvement required significantly fewer endoscopic sessions for adequate dilatation as compared to patients with muscularis propria involvement ( 1.8 vs. $6.2 ; \mathrm{P}=0.0002$ ). Patients with increased esophageal wall thickness required more endoscopic sessions for dilatation $(\mathrm{r}=0.737) \mathrm{P}=0.00004$. Three patients required

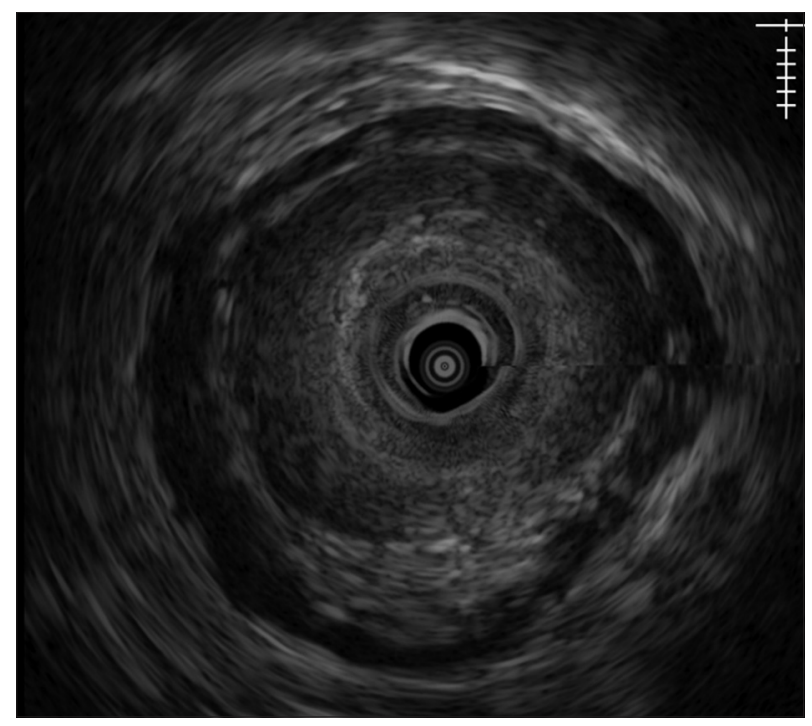

Figure 1 Miniprobe endoscopic ultrasound in a patient with a peptic stricture, showing mucosal involvement 


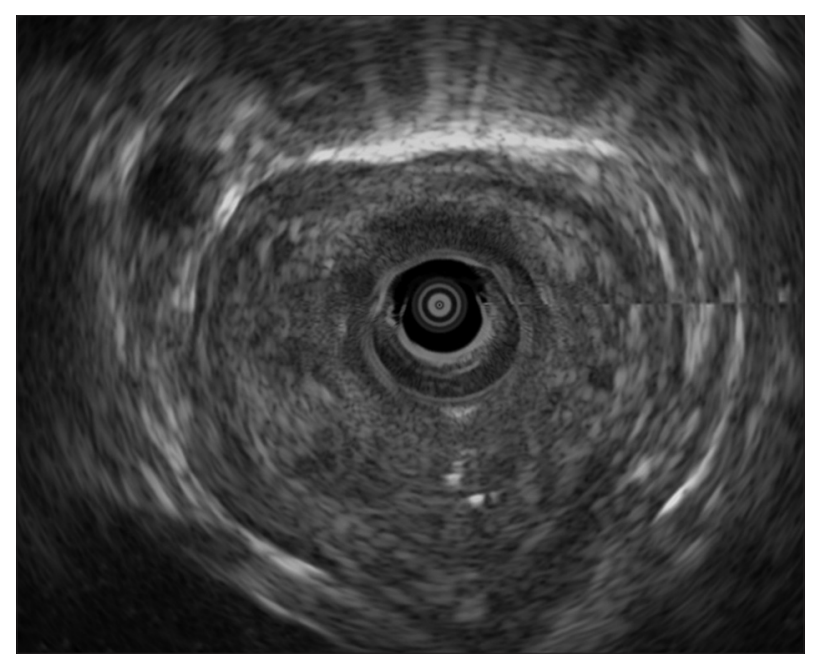

Figure 2 Miniprobe endoscopic ultrasound in a patient with a corrosive stricture, showing mucosal and submucosal involvement

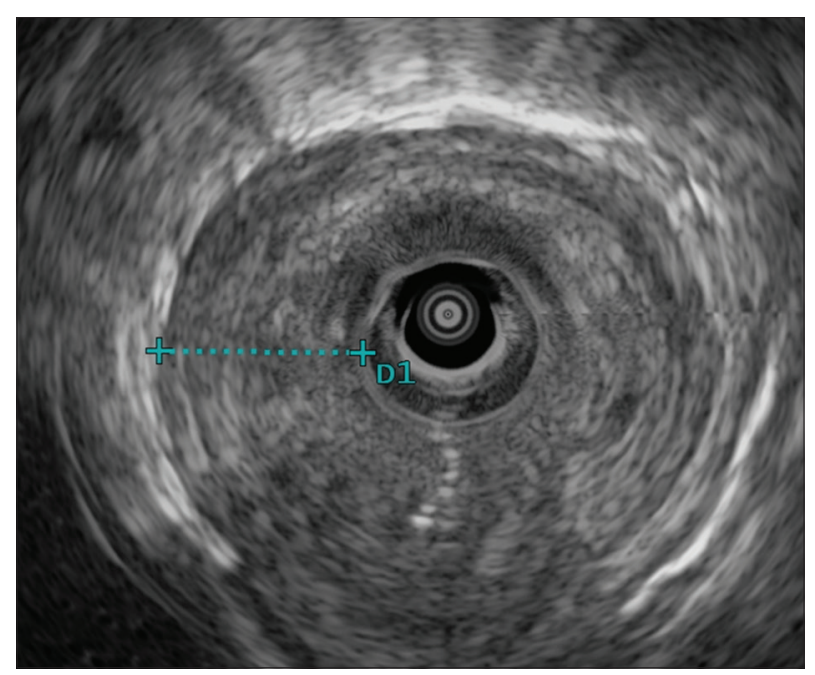

Figure 3 Miniprobe endoscopic ultrasound in a patient with a corrosive stricture, showing muscularis propria involvement

surgery for refractory strictures and all had involvement of muscularis propria. All these patients had long and thick strictures: the stricture length was $7.1,4.8$ and $7.1 \mathrm{~cm}$ and the maximum esophageal wall thickness was 11.8, 11.2 and $12.9 \mathrm{~mm}$, respectively. Similarly, symptomatic recurrence was observed in 3 patients, all of whom had involvement of muscularis propria. No significant complications of either the EUS procedure or endoscopic dilatation were observed in any patient.

\section{Discussion}

Endoscopic dilatation is the preferred treatment modality for the management of benign esophageal strictures $[2,3]$. However, the response to endoscopic dilatation is variable,

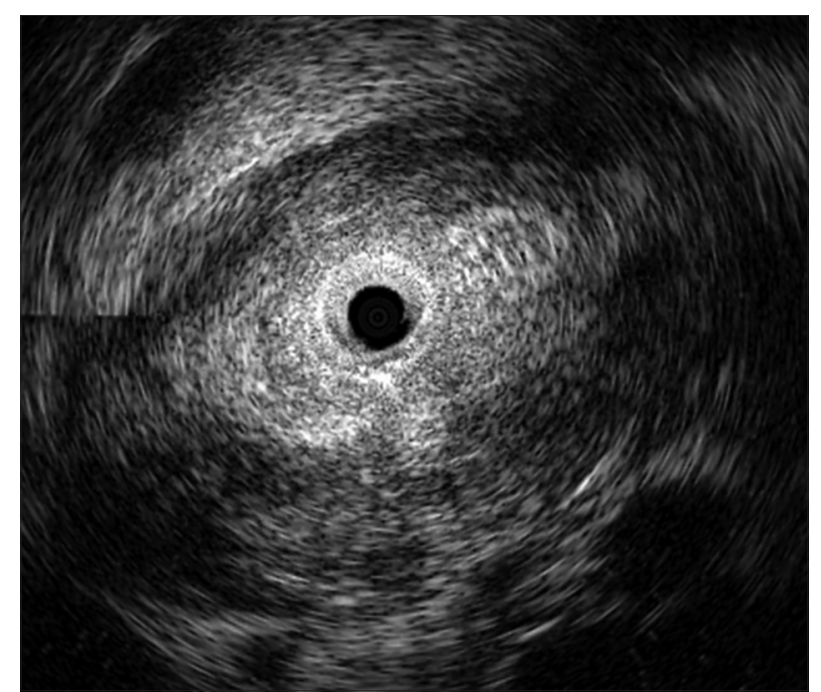

Figure 4 Miniprobe endoscopic ultrasound in a patient with a postradiation stricture, showing muscularis propria involvement

as some strictures are refractory to endoscopic dilatation and require multiple sessions or even surgery. The stricture length, as well as the esophageal wall thickness at the level of the stricture, have been found to be important predictors of the response to endoscopic dilatation $[2,3,10]$. We had previously reported that, by delineating the extent of esophageal wall involvement, EUS can predict the response to endoscopic dilatation [12]. Involvement of muscularis propria by fibrosis is an important factor that can predict the response to endoscopic dilatation: patients with muscularis propria involvement require more dilatation sessions compared to patients with involvement of mucosa and submucosa only. However, in our previous study we used a radial echoendoscope that could not be negotiated across the stricture; therefore, the evaluation of stricture was performed from the proximal end only. In the current study, we used miniprobes that could be negotiated across the stricture in all patients, allowing complete EUS examination of the stricture. The results of the current study agreed with those of our previous study that involvement of muscularis propria was an important prognostic factor determining the response to endoscopic dilatation.

We found that patients with mucosal involvement required significantly fewer endoscopic sessions for adequate dilatation, as compared to patients with submucosa and muscularis propria involvement (1.8 vs. 4.0 vs. 6.2 sessions, respectively; $\mathrm{P}=0.0008$ ). To determine whether EUS evaluation of the stricture from its proximal end is sufficient, we studied the EUS morphology of the stricture from its proximal end and compared the EUS findings obtained from the distal part of the stricture. We found that in up to $50 \%$ of patients with both submucosal and muscularis propria involvement, EUS from the proximal end of the stricture underestimated the extent of esophageal wall involvement. This confirms our hypothesis that non-negotiable conventional echoendoscopes can underestimate the esophageal wall involvement; therefore, complete evaluation of a stricture by negotiable miniprobe is needed in order to perform an adequate assessment. 
The results of our study, as well as those of previous studies, suggest that esophageal wall thickness, length of stricture and involvement of deeper layers of esophageal wall are important factors that predict the response to endoscopic dilatation. The interplay of all these factors in an individual patient needs to be studied further. Lahoti et al had previously reported that patients with short strictures but markedly increased wall thickness required more endoscopic sessions to achieve adequate dilation, in contrast to patients with long strictures and only mildly increased wall thickness, which could be easily dilated [10]. In the current study also, we found that patients with short strictures but involvement of muscularis propria required more endoscopic sessions in contrast to patients who had longer strictures but involvement of mucosa or submucosa only. Also, the majority of patients who had involvement of muscularis propria had strictures with increased wall thickness.

The small sample size, the fact that it was a single-center study, and the retrospective study design are important limitations of our study. In addition, we could not examine the impact of the extent of esophageal wall involvement on

\section{Summary Box}

\section{What is already known:}

- Benign esophageal strictures have a variable response to endoscopic dilatation

- Endoscopic ultrasound (EUS), by measuring the maximal wall thickness as well as depth of involvement, can predict the response to endoscopic dilatation

- Conventional echoendoscopes cannot be negotiated across a stricture and therefore cannot completely evaluate it

\section{What the new findings are:}

- EUS from the proximal end of a stricture underestimated the extent of esophageal wall involvement in a significant proportion of patients

- Miniprobe EUS, by completely evaluating the esophageal stricture, provided more detailed information about the extent of esophageal wall involvement

- Miniprobe EUS, by delineating the extent of wall involvement as well as measuring maximum wall thickness in benign esophageal strictures, could predict the response to endoscopic dilatation recurrence, or the need for surgery, because of the small number of patients in each group. The applicability of statistical analysis in our study is also limited for the same reason. Despite these limitations, our exploratory study confirmed the important role of the extent of esophageal wall involvement in predicting the response to endoscopic dilatation.

In conclusion, complete evaluation of esophageal strictures by negotiable miniprobe is needed to adequately assess the esophageal wall involvement. By delineating the extent of wall involvement, as well as measuring the maximum wall thickness in benign esophageal strictures, miniprobe EUS can predict the response to endoscopic dilatation.

\section{References}

1. Ravich WJ. Endoscopic management of benign esophageal strictures. Curr Gastroenterol Rep 2017;19:50.

2. Poincloux L, Rouquette O, Abergel A. Endoscopic treatment of benign esophageal strictures: a literature review. Expert Rev Gastroenterol Hepatol 2017;11:53-64.

3. Ferguson DD. Evaluation and management of benign esophageal strictures. Dis Esophagus 2005;18:359-364.

4. Patterson DJ, Graham DY, Smith JL, et al. Natural history of benign esophageal stricture treated by dilatation. Gastroenterology 1983;85:346-350.

5. Repici A, Small AJ, Mendelson A, et al. Natural history and management of refractory benign esophageal strictures. Gastrointest Endosc 2016;84:222-228.

6. Lew RJ, Kochman ML. A review of endoscopic methods of esophageal dilation. J Clin Gastroenterol 2002;35:117-126.

7. Duseja A, Chawla YK, Singh RP, Sharma TR, Kaur U, Dilawari JB. Dilatation of benign oesophageal strictures: 10 years' experience with Celestin dilators. J Gastroenterol Hepatol 2000;15:26-29.

8. Broor SL, Kumar A, Chari ST, et al. Corrosive oesophageal strictures following acid ingestion: clinical profile and results of endoscopic dilatation. J Gastroenterol Hepatol 1989;4:55-61.

9. Broor SL, Raju GS, Bose PP, et al. Long term results of endoscopic dilatation for corrosive oesophageal strictures. Gut 1993;34:1498-1501.

10. Lahoti D, Broor SL, Basu PP, Gupta A, Sharma R, Pant CS. Corrosive esophageal strictures: predictors of response to endoscopic dilation. Gastrointest Endosc 1995;41:196-200.

11. Rana SS, Bhasin DK, Sinha SK, Nagi B, Singh K. Education and imaging. Gastrointestinal: endoscopic ultrasonography (EUS) in corrosive esophageal stricture. J Gastroenterol Hepatol 2010;25:840.

12. Rana SS, Bhasin DK, Singh K. Role of endoscopic ultrasonography (EUS) in management of benign esophageal strictures. Ann Gastroenterol 2011;24:280-284.

13. Atkinson M, Ferguson R, Ogilvie AL. Management of malignant dysphagia by intubation at endoscopy. J R Soc Med 1979;72:894-897.

14. Rana SS, Sharma R, Gupta R. High-frequency miniprobe endoscopic ultrasonography for evaluation of indeterminate esophageal strictures. Ann Gastroenterol 2018;31:680-684. 